\title{
Cholangioscopy and its Role in Primary Sclerosing Cholangitis
}

\author{
Authors: \\ Brian M. Fung, ${ }^{1,2}$ M. Phillip Fejleh, ${ }^{3}$ Sooraj Tejaswi, ${ }^{4}$ *James H. Tabibiann ${ }^{2,5}$ \\ 1. Department of Medicine, Olive View-UCLA Medical Center, Los Angeles, California, \\ USA \\ 2. David Geffen School of Medicine at UCLA, Los Angeles, California, USA \\ 3. Vatche and Tamar Manoukian Division of Digestive Diseases, David Geffen School \\ of Medicine at UCLA, Los Angeles, California, USA \\ 4. Division of Gastroenterology \& Hepatology, University of California, UC Davis \\ Health, Sacramento, California, USA \\ 5. Division of Gastroenterology, Department of Medicine, Olive View-UCLA Medical \\ Center, Los Angeles, California, USA \\ *Correspondence to jtabibian@dhs.lacounty.gov
}

Disclosure:

Acknowledgements:

Received:

Accepted:

Keywords:

Citation:
The authors have declared no conflicts of interest.

Dr Fung reviewed the literature for relevant original studies and other content, designed and formatted the figures, and drafted the manuscript. Dr Tejaswi and Dr Tabibian assisted with image acquisition. Dr Fejleh, Dr Tejaswi, and Dr Tabibian critically reviewed the manuscript. All authors provided critical input and approved the final version of the manuscript. This work was completed in part through the United States National Institutes of Health (NIH) grants T32 DK007198 and UL1 TR000135.

31.01.20

19.03.20

Biliary stricture, cholangiocarcinoma (CCA), cholangioscopy-guided biopsy, cholangioscopy-guided lithotripsy, dominant stricture, peroral cholangioscopy (POC), single-operator cholangioscopy (SOC).

EMJ Hepatol. 2020;8[1]:42-53.

\section{Abstract}

Primary sclerosing cholangitis (PSC) is a cholestatic liver disease characterised by chronic inflammation and fibro-obliteration of the intrahepatic and/or extrahepatic bile ducts. It is associated with numerous hepatobiliary complications including an increased risk of malignancy (in particular, cholangiocarcinoma) and biliary tract stone formation. The evaluation of biliary strictures in patients with PSC is especially challenging, with imaging and endoscopic methods having only modest sensitivity for the diagnosis of cholangiocarcinoma, and treatment of biliary strictures poses a similarly significant clinical challenge. In recent years, peroral cholangioscopy has evolved technologically and increased in popularity as an endoscopic tool that can provide direct intraductal visualisation and facilitate therapeutic manipulation of the biliary tract. However, the indications for and effectiveness of its use in patients with PSC remain uncertain, with only a few studies performed on this small but important subset of patients. In this review, the authors discuss the available data regarding the use of peroral cholangioscopy in patients with PSC, with a focus on its use in the evaluation and management of biliary strictures and stones. 


\section{INTRODUCTION}

Primary sclerosing cholangitis (PSC) is a chronic, idiopathic, cholestatic liver disease characterised by intrahepatic and/or extrahepatic bile duct strictures and destruction. ${ }^{1,2}$ It is usually diagnosed based on a combination of persistent cholestatic liver test abnormalities and cholangiography via endoscopic retrograde cholangiopancreatography (ERCP) or magnetic resonance cholangiopancreatography showing characteristic multifocal strictures and proximal ductal dilation. ${ }^{3-5}$ PSC is also associated with many hepatobiliary complications, including an especially high risk of developing cholangiocarcinoma (CCA), estimated to be 400-1,500 times higher than in the general population. $^{6-9}$ As a result, accurate diagnosis of biliary strictures in patients with PSC is of particularly high importance. In fact, the most common indication for endoscopic intervention in patients with PSC is to evaluate and treat 'dominant strictures' seen on either initial workup for PSC or routine surveillance in patients with an established diagnosis of PSC.,10

For decades, ERCP, which relies on the use of fluoroscopy to image the biliary tree, has been the primary tool for evaluation of indeterminate strictures and other pancreaticobiliary pathology in patients with and without PSC. Other imaging modalities, such as CT and magnetic resonance cholangiopancreatography are often inadequate due to insufficient resolution, artefact, and/or the inability to acquire tissue specimen. During ERCP, intraductal brushings are often performed for cytology in order to make a diagnosis; however, the sensitivity of brushings for biliary pathology is low and limited by the poor cellular yield. This stems from the desmoplastic nature of many biliary tract neoplasms (particularly in the setting of PSC) and the inability to directly visualise a lesion while brushing, among other factors. ${ }^{11,12}$ Other endoscopic modalities, such as endoscopic ultrasound and probe-based confocal laser endomicroscopy, have been used as ancillary means of evaluating indeterminate biliary strictures; however, these modalities have their respective limitations and have generally not seen wide uptake in clinical practice. ${ }^{4}$
Over the years, another diagnostic and therapeutic method, cholangiopancreatoscopy, has been developed and refined, allowing for direct visualisation of and therapeutic manipulation within the pancreaticobiliary ducts. This technique was first described as early as 1941 in the surgery literature as an intraoperative method to exclude choledocholithiasis after cholecystectomy.13 Two decades later, a percutaneous, transhepatic approach was introduced, though this was limited by its invasiveness. ${ }^{14}$ Although the percutaneous approach is still used today, the technique has largely been replaced by a peroral approach developed in the 1970s, allowing visualisation of the biliary duct system through peroral endoscopy. Today, there are multiple peroral cholangioscopy (POC) techniques available, each with a different set of advantages and disadvantages. This review discusses the use of POC in patients with PSC, with a focus on its use in the evaluation of biliary strictures and management of biliary stones.

\section{TYPES OF CHOLANGIOSCOPY}

\section{'Mother-Baby’ Dual Operator Peroral Cholangioscopy}

The original peroral cholangioscope, developed in the 1970s, required two operators and is therefore frequently referred to as dual operator POC or 'mother-baby' POC. In this technique, two components are required: a 'mother' duodenoscope and a 'baby' cholangioscope, with one operator controlling the mother duodenoscope and one operator controlling the baby cholangioscope. To evaluate the biliary tree, the cholangioscope is threaded through the working channel of the duodenoscope which serves as a delivery mechanism. The first prototypes were limited by a lack of working channels, irrigation, and tip deflection. However, subsequent prototypes have addressed many of these problems. ${ }^{15}$ Two mother-baby POC scope systems are available today for clinical use in the USA; however, both are infrequently used due to the inconvenience of requiring two endoscopists, the fragility of the cholangioscope, and the high cost of repairs. ${ }^{16}$ 


\section{Single-Operator Peroral Cholangioscopy}

In 2005, a single-operator cholangioscopy (SOC) system called the SpyGlass ${ }^{\top M}$ Direct Visualization System (Boston Scientific Corp, Natick, Massachusetts, USA) was developed, making POC significantly more feasible and less complex, overcoming many of the shortcomings of previous methods. In this first-generation SOC system (now referred to as SpyGlass Legacy), a 10 French gauge, 230 $\mathrm{cm}$ long multichannel disposable access and delivery catheter (SpyScope ${ }^{T M}$ ) is inserted through the standard working channel of a therapeutic duodenoscope and introduced into the biliary tree with guidewire assistance after traditional ERCP-based biliary access. ${ }^{17}$ A reusable fibre optic probe (SpyGlass Direct Visualization Probe) is then advanced through a $0.9 \mathrm{~mm}$ diameter channel within the SpyScope catheter, providing 6,000-pixel images with four-way tip manoeuvrability and a $30^{\circ}$ view in each direction. The access and delivery catheter also features two $0.6 \mathrm{~mm}$ irrigation channels and a $1.2 \mathrm{~mm}$ working channel through which disposable 3 French gauge biliary biopsy forceps $\left(\right.$ SpyBite ${ }^{\mathrm{TM}}$ ) or a probe for electrohydraulic or laser-assisted lithotripsy can be inserted. The light source, video monitor, and irrigation pump are all separate components. Although the SpyGlass Legacy made POC feasible in everyday practice, there were still limitations including poor fibreoptic probe durability (rated for 8-10 uses, though lasting only 3-4 times in real life use before having to be replaced), poor image quality and field of view, a small therapeutic channel, as well as an elaborate set up with multiple separate components. ${ }^{18}$

In 2015, Boston Scientific released a secondgeneration SOC system called the SpyGlass DS Direct Visualization System, with the goal of addressing many of the shortcomings of the previous version. Notably, this new system provides better image quality (with four-times the resolution), a $60 \%$ wider field of view, improved manoeuvrability of the catheter tip, and an easier setup (reducing set-up time) compared with the previous generation system (with an integrated light source and processor). The new system also has dedicated irrigation and aspiration connections to clear the field of view and, if desired, obtain specimens..$^{19}$ In 2018, a third-generation access and delivery catheter was introduced, called the SpyScope DS II Access \& Delivery Catheter. This catheter is advertised to have even higher resolution (2.5-times that of the SpyScope DS), adjusted lighting to reduce light flare, as well as a new SpyGlass Retrieval Basket and SpyGlass Retrieval Snare for the removal of biliary stones and foreign bodies, respectively. ${ }^{20}$ There is also a newer and larger forceps, SpyBite Max, which is scheduled to be launched in the USA in 2020 and designed to facilitate acquisition of larger tissue samples.

\section{Direct Peroral Cholangioscopy}

In response to the drawbacks of mother-baby scopes, techniques using ultra-slim upper endoscopes (e.g., endoscopes originally designed for use in paediatric and transnasal applications) have been developed. In this technique, an ultra-slim upper endoscope with an external diameter ranging from 5 to $6 \mathrm{~mm}$ is advanced freehand or assisted by a guidewire or a balloon catheter to cannulate the biliary papilla. ${ }^{21,22}$ Only one operator is required, and many of the newer generation ultra-slim endoscopes provide high-definition images that can be used with narrow-band imaging (NBI) allowing detailed examination of the biliary tree (Figure 1). ${ }^{23}$ However, this technique is not without limitations. Cannulation of the bile duct and maintaining access and positioning within the common bile duct can be difficult; thus, specialised accessories and stabilisation techniques (e.g., balloon catheter, overtube balloon, guide probe of Kautz, etc.) have been developed to stabilise the endoscope. ${ }^{24-27}$ Another limitation is that the larger outer diameter of these endoscopes requires the bile duct to be dilated (limiting its use in most cases of PSC), and endoscopic sphincterotomy or sphincteroplasty with endoscopic papillary large balloon dilation pretreatment is almost always required, (which is associated with additional risks). ${ }^{28}$ 

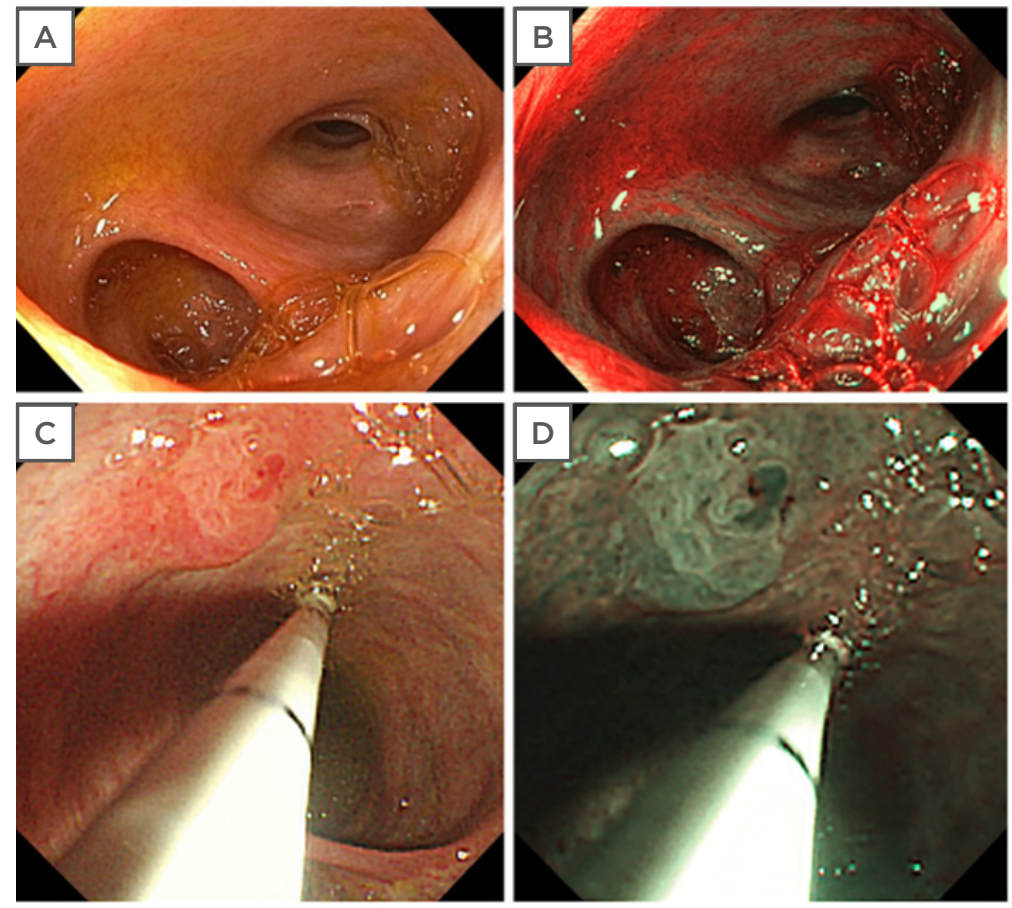

Figure 1: Narrow band imaging (NBI) assists with visualising mucosal features of the biliary tree. Noninflamed, nondysplastic biliary mucosa seen under conventional white light (A) and $\mathrm{NBI}(\mathrm{B})$. Cholangioscopic evaluation of a perihilar bile duct stricture and lesion with white light (C) and NBI (D).

Figure $1 D$ adapted from Tabibian et al. ${ }^{4}$
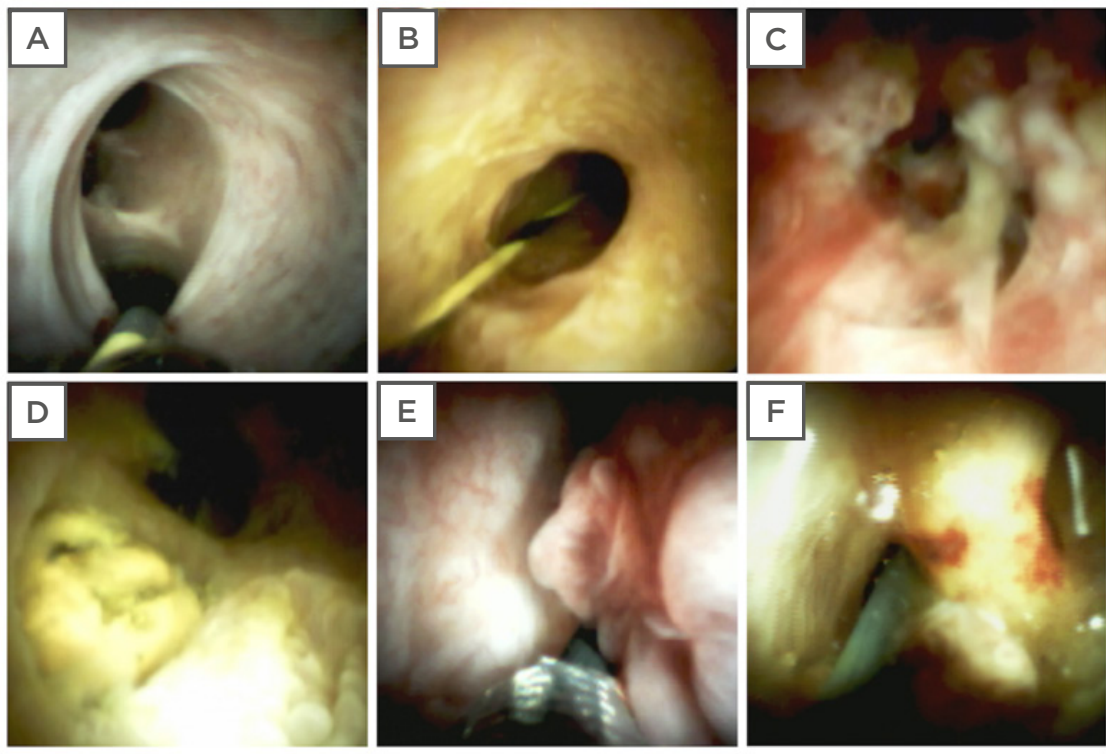

Figure 2: Applications and shortcomings of cholangioscopy illustrated with the SpyGlass ${ }^{\mathrm{TM}}$ DS Direct Visualization System.

(A) Cholangioscopy facilitates subselective ductal guidewire cannulation when conventional fluoroscopically-guided means are unsuccessful. (B) Visualisation of a benign biliary stricture in PSC. (C) Papillary fronds and abnormal vascularity of a perihilar bile duct suggestive of and subsequently proven to be CCA in a patient with PSC. (D) Biliary stone cholangioscopically identified at the cystic duct insertion which was subsequently managed with electrohydraulic lithotripsy. (E) Villous appearing biliary mucosa with papillary frond-like projections may mimic CCA; in this case, repeated SpyBite ${ }^{T M}$ sampling, intraductal brushings, and additional testing over 2 years of follow-up ruled out malignancy. (F) Abnormal intraductal erythema that can be misinterpreted as a sign of dysplasia or malignancy but was in fact caused by reactive changes related to a newly removed plastic biliary stent.

CCA: cholangiocarcinoma; PSC: primary sclerosing cholangitis. 


\section{Percutaneous Transhepatic Cholangioscopy}

Similar to direct POC, percutaneous transhepatic cholangioscopy provides direct visualisation of the biliary tree for diagnostic and therapeutic interventions. However, this technique requires significant preprocedural planning. In this technique, serial dilation of a tract is performed with subsequent tract maturation (and additional interval tract dilation) over the course of 1 week while an external biliary drainage catheter is left in place. ${ }^{29}$ Once adequate drainage is obtained and a mature tract is established, the drainage catheter can be removed over a stiff guidewire and a cholangioscope can be replaced for diagnostic and therapeutic interventions. ${ }^{29}$ This technique has several strengths. Once a percutaneous tract is established, multiple subsequent sessions can be performed. Furthermore, by using a shorter cholangioscope (which can increase manoeuvrability), areas that are difficult or impossible to reach via POC can sometimes be reached with percutaneous transhepatic cholangioscopy. Percutaneous transhepatic biliary biopsy can also be performed with high diagnostic accuracy. ${ }^{30-32}$ However, this procedure is time-consuming and has a notable risk of adverse events, including haemobilia, cholangitis, bacteraemia, bile duct injury, and tumour seeding via the sinus tract. ${ }^{33}$ As such, POC has largely replaced the use of percutaneous transhepatic cholangioscopy. ${ }^{34}$ However, for patients who are not able to tolerate a peroral endoscopic procedure (e.g., those with altered oropharyngeal or gastrointestinal anatomy), percutaneous transhepatic cholangioscopy remains a viable option. Further discussion of this technique, so far not described in the context of underlying PSC, is beyond the scope of this review but can be found elsewhere. ${ }^{35}$

\section{THE NEED FOR ENDOSCOPIC BILIARY INTERVENTION IN PRIMARY SCLEROSING CHOLANGITIS}

Because of the chronic, progressive, and variable nature of PSC, its high risk for biliary complications, and the often incomplete evaluation with imaging modalities alone, endoscopy plays a large diagnostic and therapeutic role in the management of patients with this disease. Indications for biliary endoscopy include the evaluation of dominant strictures, treatment of biliary strictures, treatment of biliary stones, intraductal foreign body retrieval (e.g., retained stent), and palliation of associated CCA. For the purposes of this concise review, and considering clinical relevance as well as available literature, the following sections focus on the evaluation of dominant strictures and treatment of biliary stones (Figure 2).

\section{Dominant Strictures}

Patients with PSC have a high propensity to develop dominant strictures, with an estimated prevalence of $36-57 \% .^{36-38}$ These strictures are defined as a stenosis of $\leq 1.5 \mathrm{~mm}$ diameter in the common bile duct or $\leq 1.0 \mathrm{~mm}$ diameter in the hepatic duct within $2.0 \mathrm{~cm}$ of the hepatic ductal confluence and are associated with poorer longterm outcomes.3,36,39 In a 25-year longitudinal study of 128 patients with PSC, the mean survival of patients with dominant strictures was almost one-half of those without dominant strictures (13.7 versus 23.0 years). ${ }^{40}$ This significant difference in survival is thought to be attributable to a combination of factors, including: 1) a high proportion of dominant strictures contain CCA;6,7 2) the lack of identifiable predictors for identifying CCA in patients with PSC;7,9 3) the lack of specific symptoms in early stages; ${ }^{2}$ and 4) the aggressiveness of CCA, with one study finding $80 \%$ of patients dying after a median period of 1 year. ${ }^{6}$ For these reasons, accurate and early differentiation between benign and malignant strictures is important for patients with PSC. Dominant strictures also frequently require therapeutic endoscopic interventions. Endoscopic balloon dilation is commonly performed for benign biliary strictures, while stent placement can be considered for strictures refractory to balloon dilation or for malignant strictures (i.e., CCA). ${ }^{2}$ A recent study has found that scheduled endoscopic dilation of dominant strictures is associated with a longer median liver-transplantation-free survival time compared with on-demand endoscopic treatment (17.9 versus 15.2 years), reinforcing the importance of early intervention. ${ }^{41}$ 

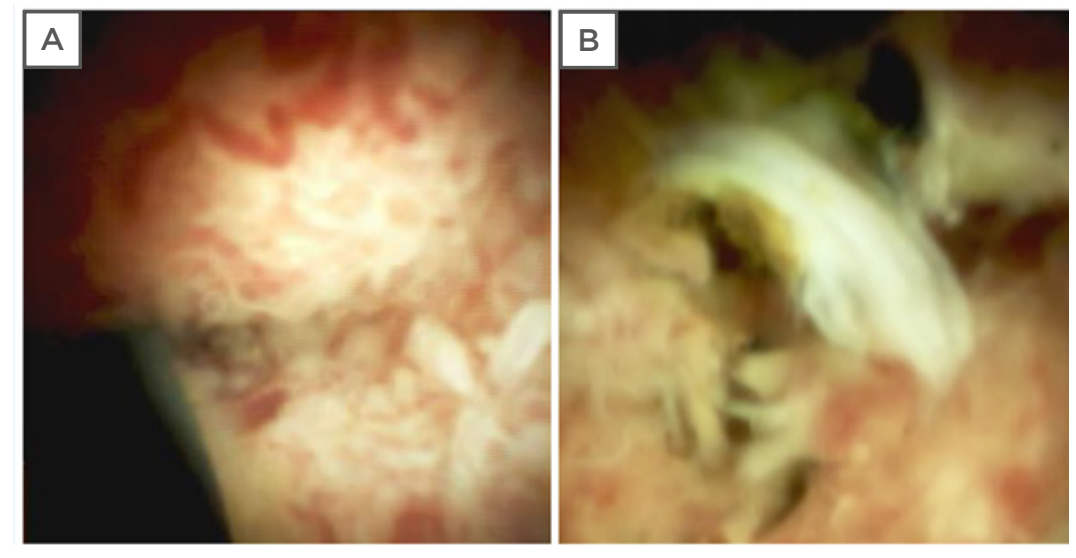

Figure 3: Identification of cholangiocarcinoma using cholangioscopy.

(A) Cholangiocarcinoma appearing as a mass growing into the lumen with associated tumour vessels in a patient without primary sclerosing cholangitis. (B) Cholangiocarcinoma appearing as an irregular growth with exudates, mucosal erythema, and luminal narrowing, without apparent classic tumour vessels in a patient with primary sclerosing cholangitis.

A classification system of extrahepatic PSC phenotypes called the Edmonton Classification has also been proposed as a way to describe and help providers with the management of dominant strictures in PSC. ${ }^{42}$

\section{Biliary Stone Disease}

Patients with PSC appear to have a high incidence of biliary stones frequently requiring endoscopic biliary intervention. Two retrospective studies of patients with PSC found that over $51 \%$ and $56 \%$ of patients with PSC who underwent ERCP and cholangioscopy, respectively, had a stone. ${ }^{43,44}$ Furthermore, one of the studies found that one-third of stones were missed on cholangiography. ${ }^{44}$ Unlike the general population in whom extrahepatic bile duct stones are relatively common and intrahepatic bile duct stones are rare, patients with PSC appear to develop stones in both locations with relatively high frequency, ${ }^{45}$ thus making stone extraction with conventional ERCP more difficult in many cases (particularly for intrahepatic stones).

\section{CHOLANGIOSCOPY IN PRIMARY SCLEROSING CHOLANGITIS}

\section{Evaluation of Indeterminate Strictures}

Over the years, endoscopists have found that POC can be helpful in the evaluation of indeterminate strictures. ${ }^{46-49}$ Summarised here are the various aspects of POC as reported in the published literature. However, the available literature must be interpreted with caution, as many studies have considerable limitations (e.g., false-negative classification, lack of comparisons to a gold standard such as pathology, limited duration of followup, etc.) and/or only a small proportion of their respective samples comprised patients with PSC.

\section{Cholangioscopic Visual Assessment}

Visual assessment of a stricture using POC has previously been suggested as a sensitive method for diagnosing malignancy in patients without PSC. ${ }^{50}$ For patients with PSC, the use of visual assessment is more unclear. Due to the very nature of the disease, patients with PSC often have significant inflammation and fibrosis of the bile ducts (Figure 3 ), limiting the ability to transverse and adequately access biliary strictures. ${ }^{51}$ Visual assessment in patients 
with PSC is also more difficult than in patients without PSC, as it is more difficult to evaluate strictures arising in a background of ductal inflammation and scarring (which itself can mimic changes of (CA) as compared to normal biliary epithelium (where CCA is generally a focal problem without the added diagnostic and therapeutic challenges of a diffusely diseased biliary tree). ${ }^{52}$ A prospective study with 47 patients with PSC found that visual assessment during POC was unable to distinguish between benign and malignant strictures. ${ }^{53}$ However, it should be noted that the vast majority of studies have been performed with the previous generation of POC, which was limited by a suboptimal image quality; current generation digital SOC (i.e., SpyGlass DS) offers superior views, which may aid in both visual diagnosis and targeting biopsies. A prospective trial is currently underway with the aim of evaluating the performance of SpyGlass DS in the diagnosis and early detection of CCA in patients with PSC. ${ }^{54}$

The utility of cholangioscopic assessment in PSC may also vary based on the specific type of CCA. One study reported that PSC has two distinct pathways of carcinogenesis, one of which has been classified as the intestinal type (a subtype that is unique to PSC and is not reported to occur in the absence of PSC), and the other is called the classical type. ${ }^{55}$ The intestinal type is predominantly seen in the hilum or secondary biliary radicles, making them well within reach of the cholangioscope. These tumours also have a distinct morphology, characterised by intraductal papillary growths with associated mucinous nodules, a pattern that is seen in cases of intraductal papillary neoplasms of the bile ducts, and can be recognised by experienced cholangioscopists. Furthermore, this type of CCA has a distinct immunohistochemical pattern that can help differentiate it from active inflammation, allowing one to overcome interobserver (both endoscopist and pathologist) variability and reduce uncertainty in the diagnosis of CCA in the setting of active inflammation or reactive mucosal changes secondary to previous stenting or balloon dilation of strictures. Future studies can determine if this type of cancer or premalignant changes can be identified based on visual assessment and immunohistochemical staining of SOC-directed biopsies. The second type of CCA described in PSC is the classical nonintestinal type that is more invasive and more difficult to detect by cholangioscopy, and therefore has a worse prognosis than intestinal type CCA. This too requires further research with the improved generation of cholangioscopes and larger biopsy forceps.

\section{Cholangioscopically-Guided Biopsies}

A systematic review and meta-analysis of 15 studies found the sensitivity and specificity of cholangioscopy-directed biopsies in all-comers (i.e., not limited to patients with PSC) to be 71.9\% (95\% confidence interval [Cl]: 66.1-77.1\%) and $99.1 \%$ (95\% Cl: 96.9-99.9\%), respectively, with a positive and negative likelihood ratio of 18.1 (95\% Cl: 9.1-35.8) and 0.3 (95\% Cl: 0.2-0.4), respectively. ${ }^{46}$ Summary receiver operating characteristic curves showed an area under the curve of 0.98, concluding that cholangioscopydirected biopsies can be useful in the evaluation of indeterminate biliary strictures. Other systematic reviews have found similarly positive results. ${ }^{47-49}$ However, the majority of these studies have not evaluated the effectiveness of POC in patients with PSC, a subset of patients with a particularly high rate of malignancy, a high rate of biliary stones, and particularly difficult biliary anatomy (caused by the prevalence of strictures making passage of the cholangioscope into the bile duct particularly challenging). ${ }^{56}$ The following section describes the limited available data on the use of POC in patients with PSC.

In 2006, Awadallah et al. ${ }^{44}$ published the first series of patients with PSC undergoing POC for the evaluation of dominant strictures and cholangioscopy-directed stone therapy. In this series of 41 patients, the authors found that POC-guided biopsies appeared to be helpful in excluding biliary malignancy (and provided a high rate of adequate tissue samples), but the study was limited by the small number of patients with a diagnosis of CCA $(n=1)$. Over the past decade, additional studies have been performed, showing promising results. A study by Tischendorf et al. ${ }^{57}$ found that POC was superior to ERCP for detecting malignancy in terms of its specificity (93\% versus 51\%), accuracy ( $93 \%$ versus 55\%), positive predictive 
value (79\% versus 29\%), negative predictive value ( $97 \%$ versus $84 \%$ ), and a trend towards better sensitivity (92\% versus $66 \%$, though this was not statistically significant). Another study supporting the use of $\mathrm{POC}$ in patients with PSC found that in 21 patients who underwent POC prior to liver transplantation, POC during a second ERCP improved the sensitivity and specificity (100\% and 97\%, respectively) for detection of CCA or high-grade dysplasia after an initial ERCP with conventional brush cytology. ${ }^{58}$ A systematic review with metaanalysis of ERCP-based modalities for the diagnosis of CCA in PSC found that SOC with targeted biopsies appeared to be the most accurate ERCP-based modality for diagnosing CCA in PSC, with a pooled sensitivity and specificity of $65 \%$ (95\% Cl: $35-87 \%$ ) and $97 \%$ (95\% Cl: 87-99\%), respectively, for the diagnosis of CCA. ${ }^{59}$ Furthermore, Kalaitzakis et al. ${ }^{56}$ found that sensitivity, specificity, and accuracy of POC for malignancy may be similar in patients with and without PSC (50\% versus 55\%, $100 \%$ versus $97 \%$, and $88 \%$ versus $80 \%$, respectively).

Other studies have found more modest outcomes for the use of POC. ${ }^{60}$ In a retrospective cohort study of 92 patients, of which 36 patients had PSC, SOC-guided biopsy combined with cytology and fluorescent in situ hybridisation demonstrated statistically significant improvement in sensitivity compared to conventional brush cytology alone (71.4\% versus 44.7\%; $\mathrm{p}=0.03) .{ }^{61}$ However, a similar improvement was not seen when restricting the analyses to the subset of patients with PSC (63.6\% versus $50.0 \%$; $p=1$ ). Moreover, a recent retrospective single-centre study of patients who underwent $\mathrm{POC}$ for indeterminate strictures (of which $40 \%$ had PSC) found that the diagnostic accuracy of POC was inferior to brush cytology and had low impact on patient management. ${ }^{62}$ A prospective study of 30 patients undergoing POC with NBI found that NBI use lead to a $48 \%$ increase in suspicious lesions biopsied, but did not improve the dysplasia detection rate. ${ }^{63}$ Additional studies, including studies with the soon-to-be launched SpyBite Max forceps, are needed to better characterise the performance characteristics of POC.

\section{Considerations in and Limitations of Cholangioscopy in the Evaluation of Indeterminate Strictures}

The utility of POC is also dependent on its success rate and ease of use. In a retrospective study of 165 patients undergoing SOC (of which 16 patients had PSC), it was reported that while POC appeared to be useful for the evaluation of indeterminate biliary lesions and difficult biliary stones in patients without PSC, the technique was associated with a lower procedure success rate (59\% versus $92 \%$ ) and lower rate of bile duct cannulation ( $82 \%$ versus $97 \%$ ) in patients with PSC compared to patients without PSC, as alluded to earlier. ${ }^{64}$ POC also increases procedure times, with one study finding the mean total procedure time of ERCP plus POC to be 45 minutes, of which 20 minutes was spent on POC. ${ }^{52}$ While the increased procedure time and additional equipment increases costs, it remains unclear whether POC leads to cost savings in the long run; one study at two Belgian academic hospitals found the use of POC for stricture diagnosis to decrease the number of procedures by $31 \%$ and costs by $5 \%$ compared with the use of ERCP alone. ${ }^{65}$ However, it should be noted that these studies report on the older legacy system, and set-up time for the newer SpyGlass DS system is significantly shorter.

Despite the aforementioned limitations and suggested modest sensitivity for malignancy, POC may also play other roles in patients with PSC. In a study on the impact of SOC on patient outcomes, SOC was noted to lead to changes in the management of nine out of 13 patients (69\%) with PSC (despite having a moderate sensitivity), and helped to avoid unnecessary hepatobiliary resection in seven patients. ${ }^{66}$ The use of POC also appears to facilitate obtaining greater quantities of tissue specimen ${ }^{63,67}$ and can provide a more accurate diagnosis of inflammatory changes than brush cytology. ${ }^{67}$

\section{Cholangioscopic Treatment of Biliary Stones}

POC is now commonly used for the treatment of biliary stones. A recent systematic review and meta-analysis of 24 studies found the rate of stone clearance with POC to be $94.3 \%$ (95\% $\mathrm{Cl}$ : $90.2-97.5 \%)$, with $71.1 \%$ of patients achieving 
stone clearance in a single session. ${ }^{68}$ Another systematic review and meta-analysis of 33 studies found a similarly high stone clearance rate of $88 \%$ (95\% Cl: $85-91 \%$ ), and the authors concluded that POC is a safe and effective method for the treatment of bile duct stones when conventional methods have failed. ${ }^{47}$ However, the aforementioned studies are not specific to patients with PSC.

Data on the performance and outcomes of POC for the treatment of biliary stones in patients with PSC are limited. In a prospective study of 41 patients who underwent POC to evaluate dominant strictures or stones, 23 (56\%) patients had stones, of which seven (30\%) were missed with cholangiography and only detected by POC. ${ }^{44}$ Seven of nine (78\%) patients who underwent POC-directed lithotripsy had complete clearance while only three of eight (38\%) patients who underwent conventional methods of stone extraction had complete clearance. In another prospective study of 32 patients (with and without PSC) who underwent POC-directed lithotripsy, four of eight (50\%) patients with PSC had stones detected by POC that were missed by cholangiography, and six of eight (75\%) patients with PSC had complete stone clearance (two had partial clearance). ${ }^{69}$ Of the four patients with recurrent stones, three had PSC. Based on the limited data available, POC-directed lithotripsy appears to be helpful for patients with PSC (and possibly cost-effective), ${ }^{65}$ though more data are needed before any definitive guidance can be provided.

\section{Treatment of Biliary Strictures and Cholangiocarcinoma}

In recent years, the use of POC has been described for the treatment of biliary strictures and malignancy (which, as previously stated, is more frequent in patients with PSC). However, data are primarily limited to case report descriptions, and there are no available studies evaluating its effectiveness specifically in patients with PSC. Nevertheless, discussed below are several recently described indications.

Biliary tract obstruction is traditionally treated with balloon dilation and/or stent placement via ERCP, with the former favoured for benign strictures and the latter for malignant strictures. ${ }^{70}$ However, either of these approaches can sometimes be challenging because of the inability to pass a guidewire through the obstructed segment and into a target duct. Bokemeyer et al. ${ }^{71}$ recently noted that POC appeared to be helpful in selective guidewire placement, especially across benign strictures. ${ }^{71}$ In several reports, POC has also been shown to be helpful in the removal of intraductal foreign bodies (e.g., retained stents). ${ }^{72-74}$ In addition, POC may play a role in radiofrequency ablation (RFA) of biliary malignancies. RFA is a technique that delivers thermal energy to induce local tissue necrosis, typically performed using ERCP guidance, and has been reported to potentially improve survival in patients with malignant strictures. ${ }^{75}$ However, it is also associated with considerable adverse events (up to $62 \%$ ), including significant bleeding, injury to adjacent vascular structures, and perforation, which may be more likely to occur when RFA is performed outside the tumour margin (which can sometimes be difficult to approximate with ERCP alone).$^{76}$ By improving localisation of the malignant stricture, POC can help increase the safety and efficacy of RFA. ${ }^{77-79}$

\section{RISKS OF CHOLANGIOSCOPY}

POC appears to be a relatively safe procedure with an adverse event rate similar to conventional ERCP alone. In a systematic review and metaanalysis consisting of 45 studies from 2000 to 2016, the pooled adverse event rate for all patients undergoing POC was $7 \%(95 \% \mathrm{Cl}$ : 6-9\%), with an estimated severe adverse event rate of $1 \%(95 \% \mathrm{Cl}: 1-2 \%) .47$ This is comparable to the adverse event rate for ERCP alone; a recent systematic survey estimated an ERCP adverse event rate of $6.85 \%$ (95\% Cl: $6.46-7.24 \%)$ and a severe adverse event rate of $1.67 \%$ (95\% $\mathrm{Cl}$ : 1.47-1.87\%). ${ }^{80}$ However, several individual studies have also reported significantly higher adverse event rates with the use of POC, the reasons for which are unclear. ${ }^{81,82}$ The most common adverse events are cholangitis (4\%), pancreatitis (2\%), and perforation (1\%) (Table 1). ${ }^{47,56,71,81-86}$ 


\section{Occurrence rate (\%)}

\begin{tabular}{ll}
\hline Common & \\
Acute cholangitis & $0.0-11.0^{47,56,71,81-86}$ \\
Acute pancreatitis & $2.0-8.9^{47,56,71,81,8}$ \\
Bacteraemia & $8.8^{83}$ \\
Bleeding & $0.0-3.3^{71,81,82,84-8}$ \\
Abdominal pain & $23.8^{81}$ \\
Rare & \\
Perforation & \\
Cardiopulmonary or sedation-related & $0.4-1.0^{47,82,84,85}$ \\
Air embolism & $0.5^{82}$ \\
$\mathrm{CO}_{2}$ embolism & $<1.0$ \\
\end{tabular}

In addition, POC appears to increase the risk of cholangitis, but the risk may be significantly reduced with peri-procedural administration of antibiotic prophylaxis. ${ }^{83,84}$ In the literature, there are also reports of air embolism (caused by the high solubility and reabsorption of air); thus, $\mathrm{CO}_{2}$ insufflation can be used instead (though case reports of $\mathrm{CO}_{2}$ emboli with uncontrolled gas insufflation can also be found in the literature). ${ }^{87,88}$ Sufficiently large papillary access (e.g., by maximum-incision papillotomy or endoscopic papillary large balloon dilation) may also reduce the risks of air/gas embolism; however, this has not been proven.

While the adverse event rate for POC appears to be similar to conventional ERCP in pooled analyses, it remains controversial whether $P O C$ increases the risk of adverse events in patients with PSC. Because strictures often prevent adequate drainage of postcontrast injection as well as the higher frequency of biliary sphincterotomy, patients with PSC have a high risk of cholangitis post-ERCP. ${ }^{89}$ However, whether POC increases the risk of cholangitis over conventional ERCP is unknown.
In a retrospective study of 92 patients (of which 36 patients had PSC) undergoing SOC, there was no difference in the rates of overall adverse events ( $14.0 \%$ versus $23.2 \%$; $p=0.27$ ) or infection (3.0\% versus $4.0 \%$; $p=0.83$ ) in patients with and without PSC. ${ }^{61}$ Furthermore, this study also found that post-ERCP abdominal pain occurred more frequently in patients without PSC compared to patients with PSC (12.0\% versus $0.0 \% ; \mathrm{p}=0.02$ ). A separate retrospective study of 341 patients (of which 12 patients had PSC) also found the rate of adverse events to be similar for patients with and without PSC. ${ }^{90}$

\section{CONCLUSION}

$\mathrm{POC}$ is an endoscopic technique which provides direct visualisation and the ability to perform therapeutic interventions within the biliary tree. In recent decades, it has been found to be a safe and effective method in the evaluation of indeterminate strictures and management of difficult to reach biliary stones. However, its usefulness in patients with PSC is unclear; additional studies on the use of this endoscopic technique in patients with PSC are needed. 


\section{References}

1. Lazaridis K, LaRusso N. Primary sclerosing cholangitis. N Engl J Med. 2016;375(12):1161-70.

2. Fung BM, Tabibian JH. Biliary endoscopy in the management of primary sclerosing cholangitis and its complications. Liver Res. 2019;3(2):10617.

3. Aabakken $L$ et al. Role of endoscopy in primary sclerosing cholangitis: European Society of Gastrointestinal Endoscopy (ESGE) and European Association for the Study of the Liver (EASL) clinical guideline. Endoscopy. 2017;49(6):588608

4. Tabibian JH et al. Advanced endoscopic imaging of indeterminate biliary strictures. World J Gastrointest Endosc. 2015:7(18):1268-78.

5. Lindor $\mathrm{K}$ et al. ACG clinical guideline: primary sclerosing cholangitis. Am J Gastroenterol. 2015;110(5):646-59.

6. Boonstra $\mathrm{K}$ et al. Populationbased epidemiology, malignancy risk, and outcome of primary sclerosing cholangitis. Hepatology. 2013;58(6):2045-55.

7. Burak $\mathrm{K}$ et al. Incidence and risk factors for cholangiocarcinoma in primary sclerosing cholangitis. Am J Gastroenterol. 2004;99(3):523-6.

8. Claessen $\mathrm{M}$ et al. High lifetime risk of cancer in primary sclerosing cholangitis. J Hepatol. 2009;50(1):158-64.

9. Chalasani $\mathrm{N}$ et al. Cholangiocarcinoma in patients with primary sclerosing cholangitis: a multicenter case-control study. Hepatology. 2000;31(1):7-11.

10. European Association for the Study of the Liver. EASL clinical practice guidelines: management of cholestatic liver diseases. J Hepatol. 2009;51(2):23767.

11. Venu R et al. Self-expandable metal stents for malignant gastric outlet obstruction: a modified technique. Endoscopy. 1998;30(6):553-8.

12. Mansfield $\mathrm{J}$ et al. A prospective evaluation of cytology from biliary strictures. Gut. 1997:40(5):671-7.

13. Mclver M. An instrument for visualizing the interior of the common duct at operation: preliminary note. Surgery. 1941:9(1):112-4

14. Shore J, Lippman H. A flexible choledochoscope. Lancet. 1965;1(7397):1200-1.

15. Franzini T et al. Advances in therapeutic cholangioscopy. Gastroenterol Res Pract. 2016;2016:5249152.

16. Erim T et al. Cholangioscopy: the biliary tree never looked so good! Curr Opin Gastroenterol. 2013;29(5):501-8.

17. Chen Y. Preclinical characterization of the Spyglass peroral cholangiopancreatoscopy system for direct access, visualization, and biopsy. Gastrointest Endosc. 2007;65(2):303-11.

18. Ayoub F et al. Cholangioscopy in the digital era. Transl Gastroenterol Hepatol. 2018;3:82.
19. Boston Scientific Corporation. SpyGlass ${ }^{T M}$ DS Direct Visualization System. 2015. Available at: https://www. bostonscientific.com/content/dam/ bostonscientific/endo/portfolio-group/ SpyGlass\%20DS/SpyGlass-DS-SystemBrochure.pdf. Last accessed: 31 March 2020.

20. Boston Scientific Corporation. SpyGlass ${ }^{T M}$ DS II Direct Visualization System. 2019. Available at: https://www. bostonscientific.com/content/dam/ bostonscientific/endo/portfolio-group/ SpyGlass\%20DS/SpyGlass-DS-Systemebrochure.pdf. Last accessed: 31 March 2020.

21. Larghi A, Waxman I. Endoscopic direct cholangioscopy by using an ultra-slim upper endoscope: a feasibility study. Gastrointest Endosc. 2006;63(6):853-7.

22. Itoi T et al. Current status of direct peroral cholangioscopy. Dig Endosc. 2011;23(Suppl 1):154-7.

23. Parsi M. Direct peroral cholangioscopy. World J Gastrointest Endosc. 2014;6(1):15.

24. Choi HJ et al. Overtube-balloon-assisted direct peroral cholangioscopy by using an ultra-slim upper endoscope (with videos). Gastrointest Endosc 2009;69(4):935-40.

25. Itoi T et al. Diagnostic and therapeutic peroral direct cholangioscopy in patients with altered $\mathrm{Gl}$ anatomy (with videos). Gastrointest Endosc. 2012;75(2):441-9.

26. Beyna T et al. A new anchoring technique for accessing the bile duct during direct peroral cholangioscopy using the guide probe of Kautz. Endoscopy. 2012;44(Suppl 2):E372-3.

27. Parsi $M$ et al. Diagnostic and therapeutic direct peroral cholangioscopy using an intraductal anchoring balloon. World $J$ Gastroenterol. 2012;18(30):3992-6.

28. Ishida $Y$ et al. Types of peroral cholangioscopy: how to choose the most suitable type of cholangioscopy. Curr Treat Options Gastroenterol. 2016;14(2):210-9.

29. Ahmed $O$ et al. Biliary interventions: tools and techniques of the trade, access, cholangiography, biopsy, cholangioscopy, cholangioplasty, stenting, stone extraction, and brachytherapy. Semin Intervent Radiol 2016;33(4):283-90.

30. Tibana T et al. The role of percutaneous transhepatic biliary biopsy in the diagnosis of patients with obstructive jaundice: an initial experience. Radiol Bras. 2019;52(4):222-8

31. Li Z et al. Value of percutaneous transhepatic cholangiobiopsy for pathologic diagnosis of obstructive jaundice: analysis of 826 cases. Acta Radiol. 2017;58(1):3-9.

32. Li TF et al. Percutaneous transhepatic cholangiobiopsy to determine the pathological cause of anastomotic stenosis after cholangiojejunostomy for malignant obstructive jaundice. Clin Radiol. 2014;69(1):13-7.
33. Oh HC et al. Analysis of percutaneous transhepatic cholangioscopy-related complications and the risk factors for those complications. Endoscopy. 2007;39(8):731-6

34. Choi JH, Lee SK. Percutaneous transhepatic cholangioscopy: does its role still exist? Clin Endosc. 2013;46(5):529-36.

35. Ahmed $\mathrm{S}$ et al. Percutaneous transhepatic cholangioscopy. Tech Vasc Interv Radiol. 2015;18(4):201-9.

36. Stiehl A et al. Development of dominant bile duct stenoses in patients with primary sclerosing cholangitis treated with ursodeoxycholic acid: outcome after endoscopic treatment. J Hepatol. 2002;36(2):151-6.

37. Tischendorf $\mathrm{J}$ et al. Characterization, outcome, and prognosis in 273 patients with primary sclerosing cholangitis: a single center study. Am J Gastroenterol. 2007;102(1):107-14.

38. Björnsson $\mathrm{E}$ et al. Dominant strictures in patients with primary sclerosing cholangitis Am J Gastroenterol. 2004;99(3):502-8.

39. Hilscher $M$ et al. Dominant strictures in primary sclerosing cholangitis: a multicenter survey of clinical definitions and practices. Hepatol Commun. 2018;2(7):836-44.

40. Chapman $\mathrm{MH}$ et al. Cholangiocarcinoma and dominant strictures in patients with primary sclerosing cholangitis; a 25-year single centre experience. Eur J Gastroenterol Hepatol. 2012;24(9):1051-

41. Rupp C et al. Effect of scheduled endoscopic dilatation of dominant strictures on outcome in patients with primary sclerosing cholangitis. Gut. 2019;68(12):2170-8.

42. Sandha $\mathrm{G}$ et al. A cholangioscopybased novel classification system for the phenotypic stratification of dominant bile duct strictures in primary sclerosing cholangitis-the Edmonton classification. J Can Assoc Gastroenterol. 2018;1(4):174-80.

43. Gluck M et al. A twenty-year experience with endoscopic therapy for symptomatic primary sclerosing cholangitis. J Clin Gastroenterol. 2008;42(9):1032-9.

44. Awadallah $\mathrm{N}$ et al. Is there a role for cholangioscopy in patients with primary sclerosing cholangitis? Am Gastroenterol. 2006;101(2):284-91.

45. Dodd $\mathrm{G}$ et al. Bile duct calculi in patients with primary sclerosing cholangitis. Radiology. 1997;203(2):443-7.

46. Badshah M et al. Peroral cholangioscopy with cholangioscopy-directed biopsies in the diagnosis of biliary malignancies: a systemic review and meta-analysis. Eur J Gastroenterol Hepatol. 2019;31(8):93540

47. Korrapati P et al. The efficacy of peroral cholangioscopy for difficult bile duct stones and indeterminate strictures: a systematic review and meta-analysis. Endosc Int Open. 2016;4(3):E263-75. 
48. Navaneethan $U$ et al. Comparative effectiveness of biliary brush cytology and intraductal biopsy for detection of malignant biliary strictures: a systematic review and meta-analysis. Gastrointest Endosc. 2015;81(1):168-76.

49. Sun $X$ et al. Is single-operator peroral cholangioscopy a useful tool for the diagnosis of indeterminate biliary lesion? A systematic review and meta-analysis. Gastrointest Endosc. 2015;82(1):79-87.

50. Ramchandani $M$ et al. Role of singleoperator peroral cholangioscopy in the diagnosis of indeterminate biliary lesions: a single-center, prospective study. Gastrointest Endosc. 2011;74(3):511-9.

51. Heif $M$ et al. ERCP with probe-based confocal laser endomicroscopy for the evaluation of dominant biliary stenoses in primary sclerosing cholangitis patients. Dig Dis Sci. 2013;58(7):206874.

52. Liu R et al. Peroral cholangioscopy facilitates targeted tissue acquisition in patients with suspected cholangiocarcinoma. Minerva Gastroenterol Dietol. 2014;60(2):127-33.

53. Arnelo $U$ et al. Prospective evaluation of the clinical utility of single-operator peroral cholangioscopy in patients with primary sclerosing cholangitis. Endoscopy. 2015;47(8):696-702.

54. Boston Scientific Corporation. Cholangioscopy in Primary Sclerosing Cholangitis (PSC). NCT03766035. https://clinicaltrials.gov/ct2/show/ NCT03766035.

55. Zen $Y$ et al. Two distinct pathways of carcinogenesis in primary sclerosing cholangitis: cholangiocarcinoma in PSC. Histopathology. 2011;59(6):1100-10.

56. Kalaitzakis E et al. Diagnostic utility of single-user peroral cholangioscopy in sclerosing cholangitis. Scand J Gastroenterol. 2014;49(10):1237-44.

57. Tischendorf $\mathrm{J}$ et al. Cholangioscopic characterization of dominant bile duct stenoses in patients with primary sclerosing cholangitis. Endoscopy. 2006;38(7):665-9.

58. Majeed A et al. Optimizing the detection of biliary dysplasia in primary sclerosing cholangitis before liver transplantation. Scand J Gastroenterol. 2018;53(1):56-63.

59. Njei B et al. Systematic review with meta-analysis: endoscopic retrograde cholangiopancreatography-based modalities for the diagnosis of cholangiocarcinoma in primary sclerosing cholangitis. Aliment Pharmacol Ther. 2016;44(11-12):1139-51.

60. Schramm C. Concise commentary: why cholangioscopy for indeterminate biliary strictures in PSC is still not good enough. Dig Dis Sci. 2019. [Epub ahead of print].

61. Kaura K et al. Cholangioscopy biopsies improve detection of cholangiocarcinoma when combined with cytology and FISH, but not in patients with PSC. Dig Dis Sci. 2019.
[Epub ahead of print].

62. de Vries A et al. Limited diagnostic accuracy and clinical impact of singleoperator peroral cholangioscopy for indeterminate biliary strictures. Endoscopy. 2020;52(2):107-14.

63. Azeem $\mathrm{N}$ et al. Cholangioscopy with narrow-band imaging in patients with primary sclerosing cholangitis undergoing ERCP. Gastrointest Endosc. 2014;79(5):773-9.e2.

64. Kalaitzakis E et al. Diagnostic and therapeutic utility of singleoperator peroral cholangioscopy for indeterminate biliary lesions and bile duct stones. Eur J Gastroenterol Hepatol. 2012;24(6):656-64.

65. Deprez $\mathrm{P}$ et al. The economic impact of using single-operator cholangioscopy for the treatment of difficult bile duct stones and diagnosis of indeterminate bile duct strictures. Endoscopy. 2018;50(2):109-18.

66. Prat F et al. Impact of perora cholangioscopy on the management of indeterminate biliary conditions: a multicentre prospective trial. Frontline Gastroenterol. 2019;10(3):236-43.

67. Rey J et al. Efficacy of SpyGlass( $\left.{ }^{\mathrm{TM}}\right)$ directed biopsy compared to brush cytology in obtaining adequate tissue for diagnosis in patients with biliary strictures. World J Gastrointest Endosc. 2014;6(4):137-43.

68. Jin $Z$ et al. Single-operator peroral cholangioscope in treating difficult biliary stones: a systematic review and meta-analysis. Dig Endosc. 2019;31(3):256-69.

69. Piraka $\mathrm{C}$ et al. Transpapillary cholangioscopy-directed lithotripsy in patients with difficult bile duct stones. Clin Gastroenterol Hepatol. 2007;5(11):1333-8.

70. Ponsioen $\mathrm{C}$ et al. No superiority of stents vs balloon dilatation for dominant strictures in patients with primary sclerosing cholangitis. Gastroenterology. 2018;155(3):752-9.e5.

71. Bokemeyer A et al. Digital singleoperator cholangioscopy: a useful tool for selective guidewire placements across complex biliary strictures. Surg Endosc. 2019;33(3):731-7.

72. Albert $\mathrm{J}$ et al. Peroral cholangioscopy for diagnosis and therapy of biliary tract disease using an ultra-slim gastroscope. Endoscopy. 2011;43(11):1004-9.

73. Fejleh MP et al. Cholangioscopy-guided retrieval basket and snare for the removal of biliary stones and retained prostheses. VideoGIE. 2019;4(5):232-4.

74. Bas-Cutrina F et al. Removal of a migrated biliary stent using new digital cholangioscopy retrieval devices in a transplant patient. Endoscopy. 2019;51(11):E323-4.

75. Sharaiha R et al. Impact of radiofrequency ablation on malignant biliary strictures: results of a collaborative registry. Dig Dis Sci.
2015;60(7):2164-9.

76. Mensah E et al. Radiofrequency ablation for biliary malignancies. Curr Opin Gastroenterol. 2016;32(3):238-43.

77. Ogura T et al. Evaluation of the safety of endoscopic radiofrequency ablation for malignant biliary stricture using a digital peroral cholangioscope (with videos). Dig Endosc. 2017;29(6):712-7.

78. Gunasingam N, Craig P. Cholangioscopydirected radiofrequency ablation of complex biliary cholangiocarcinoma. VideoGIE. 2019;4(5):211-3.

79. Mansilla-Vivar $\mathrm{R}$ et al. Endoluminal radiofrequency ablation with SpyGlass ${ }^{\top M}$ in the management of cholangiocarcinoma. Rev Esp Enferm Dig. 2019;111(10):803-5.

80. Andriulli $A$ et al. Incidence rates of post-ERCP complications: a systematic survey of prospective studies. Am J Gastroenterol. 2007;102(8):1781-8.

81. Lenze $F$ et al. Safety, diagnostic accuracy and therapeutic efficacy of digital single-operator cholangioscopy. United European Gastroenterol J. 2018;6(6):902-9.

82. Sethi A et al. ERCP with cholangiopancreatoscopy may be associated with higher rates of complications than ERCP alone: a single-center experience. Gastrointest Endosc. 2011;73(2):251-6.

83. Othman $M$ et al. A prospective study of the risk of bacteremia in directed cholangioscopic examination of the common bile duct. Gastrointest Endosc. 2016;83(1):151-7.

84. Turowski F et al. Diagnostic and therapeutic single-operator cholangiopancreatoscopy with SpyGlassDS ${ }^{\mathrm{TM}}$ : results of a multicenter retrospective cohort study. Surg Endosc. 2018;32(9):3981-8

85. Adler D et al. A large multicenter study analysis of adverse events associated with single operator cholangiopancreatoscopy. Minerva Gastroenterol Dietol. 2015;61(4):179-84.

86. Gerges $C$ et al. Digital single-operator peroral cholangioscopy-guided biopsy versus ERCP-guided brushing for indeterminate biliary strictures: a prospective, randomized multicenter trial (with video). Gastrointest Endosc 2019. [Epub ahead of print]

87. Romberg C. Systemic air embolism after ERCP: a case report and review of the literature (with video). Gastrointest Endosc. 2009;70(5):1043-5.

88. Hann A et al. Fatal outcome due to $\mathrm{CO}_{2}$ emboli during direct cholangioscopy. Gut. 2018;67(8):1378-9.

89. Navaneethan $U$ et al. ERCP-related adverse events in patients with primary sclerosing cholangitis. Gastrointest Endosc. 2015;81(2):410-9.

90. Bernica $J$ et al. Cholangioscopy is safe and feasible in elderly patients. Clin Gastroenterol Hepatol. 2018;16(8):1293 9.e2 